УДК 536.521 .3

\title{
ВЛИЯНИЕ СЕЛЕКТИВНОСТИ ТЕПЛОВОГО ИЗЛУЧЕНИЯ МЕТАЛЛИЧЕСКИХ СПЛАВОВ НА МЕТОДИЧЕСКИЕ ПОГРЕШНОСТИ ИХ КЛАССИЧЕСКОЙ И ДВУХЦВЕТОВОЙ КОМПЕНСАЦИОННОЙ ТЕРМОМЕТРИИ
}

\author{
Жуков Л.Ф., Д. т. н., Петренко Д.А. \\ Физико-технологический институт металлов и сплавов НАН Украины, \\ бульв. Вернадского, 34/1, 03142, Киев, Украина
}

\begin{abstract}
Встановлено значно вищі Представлено результати енергетиметрологічні характеристики, у чУстановлены значительно более высопорівнянні з відомими рішеннями, за- кие метрологические характеристики, пропонованого метода двокольорової по сравнению с известными решениями, компенсаційної пірометрії випромі- предложенного метода двухцветовой нювання металевих сплавів із ви- компенсационной пирометрии излучепадково змінною селективністю ния металлических сплавов со случайно випромінювання. Методичні похиб- изменяющейся селективностью излучеки нового методу нижчі, ніж похибки ния. Методические погрешности нового енергетичної однокольорової, а також метода ниже погрешностей энергетичеспектрального відношення пірометрії ской одноцветовой, а также спектральвипромінювання в 4,4..6,2 і 40,2 рази, ного отношения пирометрии излучения відповідно.

в 4,4..6,2 и 40,2 раза, соответственно.
\end{abstract}

\begin{abstract}
Much higher metrological characteristics, in comparison with known solutions, of proposed two-colour compensating thermometry method of metal alloys with randomly variable selectivity of radiation were determined. Methodical errors of two-colour compensating thermometry are lower than errors of energy one-colour and spectral ratio pyrometry in $4,4 \ldots 6,2$ and 40,2 times, respectively.
\end{abstract}

Библ. 3, рис. 2.

Ключевые слова: температура, металлические сплавы, двухцветовая компенсационная, энергетическая одноцветовая и спектрального отношения пирометрия излучения, коэффициент селективности излучения, относительная методическая погрешность.

В результате выполненных в работе [1] исследований и анализа обосновано также ожидаемое влияние оптических характеристик термометрируемых объектов на методические погрешности двухцветовой компенсационной и классической пирометрии излучения. В значительной мере это относится к селективности излучения термометрируемых объектов и, прежде всего, металлических сплавов. Излучательные характеристики металлов и их сплавов, в отличие, например, от их оксидов, боридов, карбидов, нитридов и силицидов в производственных условиях случайно изменяются в более широких пределах. В инертной среде и вакууме, в соответствии с законами классической электродинамики, интенсивность и спектральное распределение теплового излучения сплавов определяются их электромагнитными характеристиками, которые, в свою очередь, зависят от примесей и неметаллических включений. В наиболее характерной для металлургии обычной атмосфере, диапазон случайных изменений селективности определяется доминирующим воздействием оксидных плен, которое, в свою очередь, зависит от интенсивности и направления окислительно-восстановительных процессов. Количественно селективность излучения можно оценить соответствующим коэффициентом $k=\frac{\varepsilon_{\lambda_{1}}}{\varepsilon_{\lambda_{2}}}$, равным отношению значений излучательной способности на рабочих волнах $\lambda_{1}$ и $\lambda_{2}$. Для спадающих и воз- растающих распределений излучательной способности коэффициент селективности соответственно больше и меньше единицы. Под воздействием интерференции излучения на покрывающих металл тонких прозрачных оксидных пленах коэффициент селективности может изменяться в широких пределах, при относительно стабильном среднем уровне излучательной способности. Обычно это имеет место для сплавов в струе или потоке, на выпуске или сливе, из металлургических печей и агрегатов.

В настоящей работе представлены результаты исследований, выполненных в диапазоне $k$ от 1,000 до 1,186 , существенно перекрывающем его реальные значения для наиболее распространенных в металлургии железоуглеродистых сплавов. При $k=1$ излучение не селективно и является серым. Это имеет место, например, для сплавов, покрытых непрозрачными оксидными пленами. На рис. 1 пунктиром представлены исследованные спектральные распределения излучательной способности для граничных значений коэффициента селективности $\left(k_{\min }=1,000 ; k_{\max }=1,186\right)$ при среднем уровне излучательной способности $\varepsilon_{\text {cр }}=\varepsilon_{\text {cр н }}=0,417$, соответствующем вольфраму в вакууме с $T=1600 \mathrm{~K}$ в видимой и ближней инфракрасной областях спектра. Сплошной линией показано спектральное распределение вольфрама с $k=k_{\text {н }}=1,093$, выбранное в качестве 
настроечного для исследуемых методов пирометрии излучения. Значения излучательной способности $\varepsilon_{\lambda_{1}}$ и $\varepsilon_{\lambda_{2}}$ введены в пирометрические уравнения для классической и двухцветовой компенсационной пирометрии излучения как настроечные параметры. Использованные характеристики вольфрама представительны для железоуглеродистых сплавов, детально изучены и табулированы в литературе по оптике металлов и оптической термометрии [2]. С целью исключения влияния излучательной способности и температуры на результаты исследований, последние выполнены при $\varepsilon_{\text {ср }}=$ const и $T=$ const.

Полученные в результате сравнительных исследований закономерности влияния селективности излучения сплавов на относительные методические погрешности двухцветовой компенсационной пирометрии излучения (ДКПИ) $\left(\delta T_{\text {дкпи }}\right)$, а также классической энергетической одноцветовой на $\lambda_{1}$ и $\lambda_{2}\left(\delta T_{\lambda_{1}}\right.$ и $\left.\delta T_{\lambda_{2}}\right)$ и спектрального отношения на $\lambda_{\text {экв }}=\frac{\lambda_{1} \lambda_{2}}{\lambda_{2}-\lambda_{1}}\left(\delta T_{\text {со }}\right)$ термометрии приведе-
ны на рис. 2 .

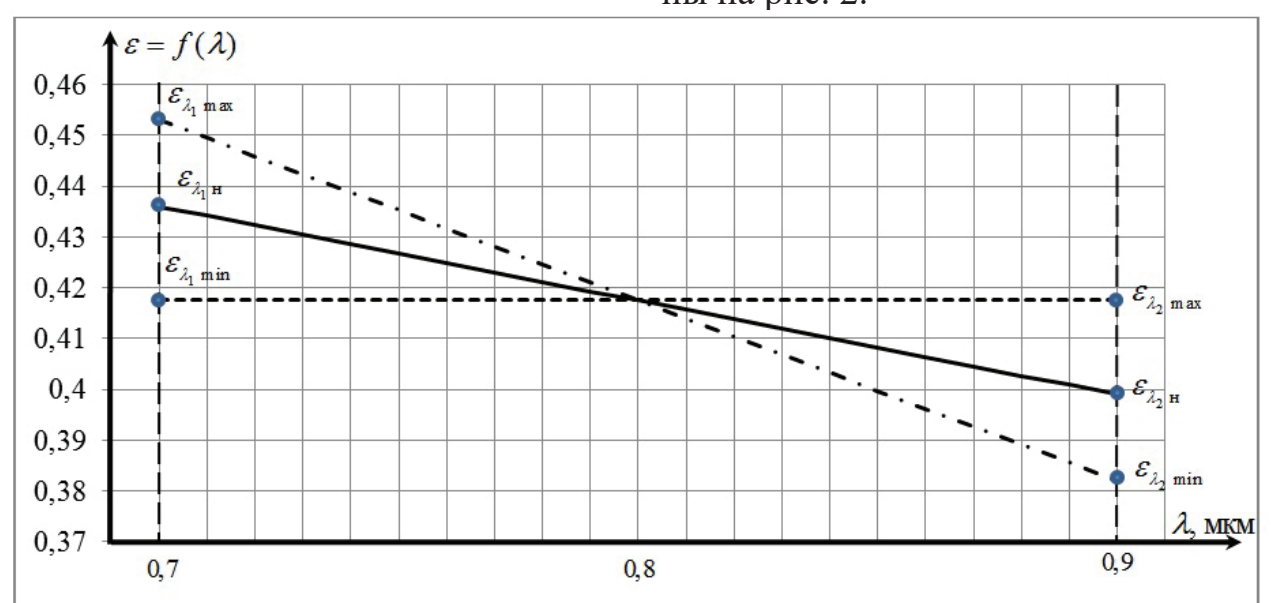

Рис. 1. Исследованные спектральные распределения излучательной способности металлических сплавов.

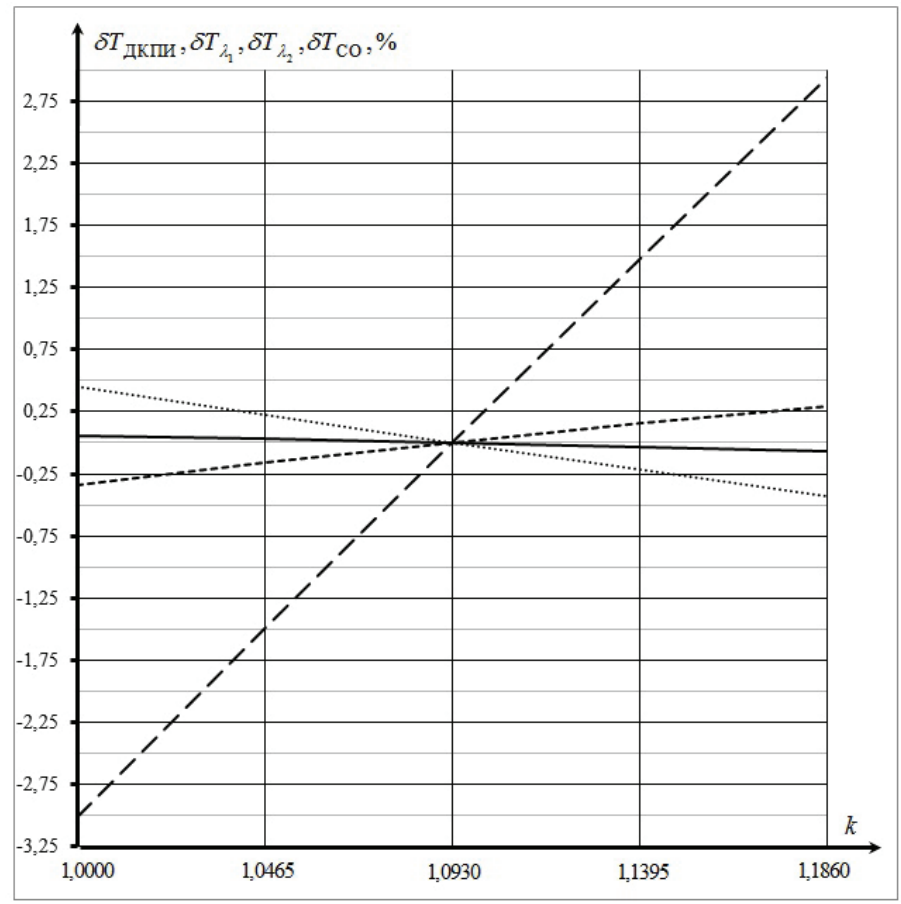

Рис. 2. Влияние селективности излучения металлических сплавов на относительные методические погрешности пирометрии излучения при $T=$ const $=1600 \mathrm{~K} \boldsymbol{u} \varepsilon_{\mathrm{cp}}=$ const $=0,417$ :

\footnotetext{
- двухцветовой компенсационной на $\lambda_{1}=0,70$ мкм и $\lambda_{2}=0,90$ мкм;

---.- одноцветовой на $\lambda_{1}=0,70$ мкм;

одноцветовой на $\lambda_{2}=0,90$ мкм;

- спектрального отночения на $\lambda_{\text {экв }}=3,15$ мкм.
} 
Для настроечного значения коэффициента селективности излучения $k_{\mathrm{H}}=1,093$ введены температурные поправки на методические погрешности двухцветовой компенсационной, а также классической энергетической и спектрального отношения пирометрии излуче-

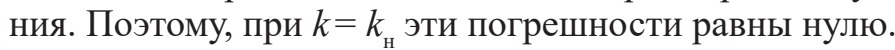

По модулю погрешности двухцветовой компенсационной, одноцветовой и спектрального отношения пирометрии излучения располагаются в следующем порядке: $\left|\delta T_{\text {дкпи }}\right|<\left|\delta T_{\lambda_{1}}\right|<\left|\delta T_{\lambda_{2}}\right| \delta T_{\text {со }} \mid$. Принцип компенсации обеспечивает минимальные погрешности ДКПИ. Погрешности нового метода ниже $\delta T_{\lambda_{1}}$ и $\delta T_{\lambda_{2}}$, так как для определения зеркальных одноцветовых температур излучения используются настроечные значения излучательной способности $\varepsilon_{\lambda_{1 н}}$ и $\varepsilon_{\lambda_{2} \text {, }}$ относительно которых отклоняются $\varepsilon_{\lambda}$ и $\varepsilon_{\lambda_{2}}$ при изменении коэффициента селективности. Причем величины этих отклонений равны по модулю и противоположны по знаку.

При увеличении $k$ от 1,000 до 1,186 погрешность ДКПИ по модулю уменьшается от 0,058 до $0,000 \%$ и затем повышается от 0,000 до $0,069 \%$. Полученная закономерность объясняется тем, что по условиям исследований введена температурная поправка, обнуляющая методическую погрешность при $k=k_{\text {н. }}$ Кроме того, с изменением коэффициента селективности излучения от минимального до максимального соответствующие в реальных условиях незначительные изменения $\varepsilon_{\lambda_{1}}$ и $\varepsilon_{\lambda}$ вызывают «однознаковые» и близкие по модулю Погрешности $\Delta S_{1}$ и $\Delta S_{2}$ для зеркальных одноцветовых температур излучения. Отличие между $\Delta S_{1}$ и $\Delta S_{2}$ определяется влиянием длины волны $\lambda_{2}$. Вследствие того, что расчет зеркальной одноцветовой температуры излучения $S_{1}$ ведется с использованием измеренной одноцветовой температуры излучения $S_{2}$, методическая погрешность которой превышает погрешность $S_{1}$ из-за влияния $\lambda_{1}>\lambda_{2}$, имеет место соотношение $\left|\Delta S_{1}^{\prime}\right|>\mid \Delta S_{2}$. С использованием выведенных из закона Вина пирометрических уравнений для зеркальных одно- и двухцветовых температур излучения [3] нами получены выражения (1-3) для абсолютных методических погрешностей $\Delta S_{1}^{\prime}, \Delta S_{2}$ и $\Delta S_{24}$. Погрешность $\Delta S_{1}$ для зеркальной одноцветовой температуры излучения на $\lambda_{1}$ определяется выражением

$$
\begin{aligned}
& \Delta S_{1}^{\prime}=S_{1 \mathrm{p}}^{\prime}-S_{1}^{\prime}=\frac{S_{2}\left(T, \varepsilon_{\lambda_{2}}\right) \cdot C_{2}}{S_{2}\left(T, \varepsilon_{\lambda_{2}}\right) \cdot \ln \left(\varepsilon_{\lambda_{2} \mathrm{H}}\right) \cdot\left(\lambda_{2}-\lambda_{1}\right)+C_{2}}- \\
& -\frac{S_{2}\left(T, \varepsilon_{\lambda_{2}}\right) \cdot C_{2}}{S_{2}\left(T, \varepsilon_{\lambda_{2}}\right) \cdot \ln \left(\varepsilon_{\lambda_{2}}\right) \cdot\left(\lambda_{2}-\lambda_{1}\right)+C_{2}},
\end{aligned}
$$

где $C_{2}=0,014388 \mathrm{~K} \cdot \mathrm{M}-$ вторая постоянная Планка;

$S_{1 \text { p }}$ - расчетная зеркальная одноцветовая температура излучения для $\varepsilon_{\lambda_{2}}$;

$S_{1}$ - правильная зеркальная одноцветовая температура излучения, которая соответствует $\varepsilon_{\lambda}$, отличному от $\varepsilon_{\lambda_{2}}$; $\mathrm{S}_{2}$ - измеренная одноцветовая температура излучения на $\lambda_{2}$.
Очевидно, что когда $\varepsilon_{\lambda_{2}}=\varepsilon_{\lambda_{2}}$, то $\Delta S_{1}^{\prime}=0$. Если $k<k_{\mathrm{H}}$, то, как видно из рис. $1, \varepsilon_{\lambda}>\varepsilon_{\lambda}$."Отсюда следует, что знаменатель вычитаемого в (1) будет больше, чем знаменатель уменьшаемого, так как $\left|\ln \left(\varepsilon_{\lambda_{2}}\right)\right|>\left|\ln \left(\varepsilon_{\lambda_{2}}\right)\right|$ и, следовательно, $\Delta S_{1}>0$. Если $k>k_{\text {н }}$, то $\varepsilon_{\lambda_{2}}<\varepsilon_{\lambda_{2}}$. В этом случае знаменатель вычитаемого в (1) будет меньше, чем знаменатель уменьшаемого, так как $\left|\ln \left(\varepsilon_{\lambda_{2}}\right)\right|<\left|\ln \left(\varepsilon_{\lambda_{2}}\right)\right|$ и $\Delta S_{1}^{\prime}<0$.

Выражение погрешности $\Delta S_{1}$ для зеркальной одноцветовой температуры излучения на $\lambda_{2}$ имеет аналогичный вид (2).

$$
\begin{aligned}
& \Delta S_{2}^{\prime}=S_{2 \mathrm{p}}^{\prime}-S_{2}^{\prime}=\frac{-S_{1}\left(T, \varepsilon_{\lambda_{1}}\right) \cdot C_{2}}{S_{1}\left(T, \varepsilon_{\lambda_{1}}\right) \cdot \ln \left(\varepsilon_{\lambda_{1} \mathrm{H}}\right) \cdot\left(\lambda_{2}-\lambda_{1}\right)-C_{2}}+ \\
& +\frac{S_{1}\left(T, \varepsilon_{\lambda_{1}}\right) \cdot C_{2}}{S_{1}\left(T, \varepsilon_{\lambda_{1}}\right) \cdot \ln \left(\varepsilon_{\lambda_{1}}\right) \cdot\left(\lambda_{2}-\lambda_{1}\right)-C_{2}},
\end{aligned}
$$

где $S_{2 \mathrm{p}}$ - расчетная зеркальная одноцветовая температура излучения для $\varepsilon_{\lambda_{1}}$;

$S_{2}$ - правильная зеркальная одноцветовая температура излучения, которая соответствует $\varepsilon_{\lambda_{1}}$, отличному от $\varepsilon_{\lambda_{1}}$; $S_{1}$ - измеренная одноцветовая температура излучения на $\lambda_{1}$.

При $\varepsilon_{\lambda_{1}}=\varepsilon_{\lambda_{1}}, \Delta S_{2}=0$. Если $k<k_{\text {н }}$, то $\varepsilon_{\lambda_{1}}<\varepsilon_{\lambda_{1}}$ (рис. 1). Из этого видно, что знаменатель первого слагаемого в (2) будет меньше по модулю, чем знаменатель второго, так как $\left|\ln \left(\varepsilon_{\lambda_{14}}\right)\right|<\left|\ln \left(\varepsilon_{\lambda_{1}}\right)\right|$. Первое слагаемое будет положительным и по модулю превысит второе (отрицательное), в результате чего $\Delta S_{2}>0$. Если $k>k_{\text {н }}$, то $\varepsilon_{\lambda}>\varepsilon_{\lambda_{1}}$. В этом случае знаменатель первого слагаемого в (2) будет больше по модулю, чем знаменатель второго, так как $\left|\ln \left(\varepsilon_{\lambda_{1}}\right)\right|>\left|\ln \left(\varepsilon_{\lambda_{1}}\right)\right|$. Первое слагаемое будет положительным и по модулю меньше второго (отрицательного), в результате чего $\Delta S_{<}<0$.

Так как $S_{1 \mathrm{p}}=S_{1}+\Delta S_{1}$ и $S_{2 \mathrm{p}}=S_{2}+\Delta S_{2}$, погрешность $\Delta S_{2 \Perp}$ для зеркальной двухцветовой температуры излучения определяется выражением (3)

$$
\Delta S_{2 \amalg}^{\prime}=S_{2 \amalg \mathrm{p}}^{\prime}-S_{2 \amalg}^{\prime}=\frac{1}{\lambda_{\text {экв }}\left(\frac{1}{\lambda_{1} \cdot S_{1 \mathrm{p}}^{\prime}}-\frac{1}{\lambda_{2} \cdot S_{2 \mathrm{p}}^{\prime}}\right)}-\frac{1}{\lambda_{\text {ЭКв }}\left(\frac{1}{\lambda_{1} \cdot S_{1}^{\prime}}-\frac{1}{\lambda_{2} \cdot S_{2}^{\prime}}\right)},
$$

где $S_{2 ц \mathrm{p}}$ - расчетная зеркальная двухцветовая температура излучения для $\varepsilon_{\lambda,}, \varepsilon_{\lambda}$;

$S_{2 \longleftarrow}$ - правильная зеркальная двухцветовая температура излучения, которая соответствует $\varepsilon_{\lambda_{1}}, \varepsilon_{\lambda_{2}}$, отличным от

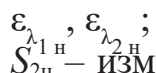
$S_{2 \Perp}^{1 н}$ - измеренная двухцветовая (спектрального отношения) температура излучения на $\lambda_{\text {экв }}$.

При $\Delta S_{1}^{\prime}>0$ и $\Delta S_{2}^{\prime}>0, \frac{1}{\lambda_{1} \cdot S_{1 \mathrm{p}}^{\prime}}<\frac{1}{\lambda_{1} \cdot S_{1}^{\prime}}$ и $\frac{1}{\lambda_{2} \cdot S_{2 \mathrm{p}}^{\prime}}<\frac{1}{\lambda_{2} \cdot S_{2}^{\prime}}$. Следовательно, $\left(\frac{1}{\lambda_{1} \cdot S_{1 \mathrm{p}}^{\prime}}-\frac{1}{\lambda_{2} \cdot S_{2 \mathrm{p}}^{\prime}}\right)<\left(\frac{1}{\lambda_{1} \cdot S_{1}^{\prime}}-\frac{1}{\lambda_{2} \cdot S_{2}^{\prime}}\right)$, а $\Delta S_{2 \text { ц }}>0$ и $\Delta T_{\text {дкпи }}>0$. Аналогично можно показать, что при $\Delta S_{1}<0$ и $\Delta S_{2}<0$, $\Delta S_{2 \Perp}<0$ и $\Delta T_{\text {дкпи }}<0$. Так как $\lambda_{\text {экв }}>\lambda_{1}$ и $\lambda_{\text {экв }}>\lambda_{2}$, то $\left|\Delta S_{2 \Perp}\right|>\left|\Delta S_{1}\right|$ 
и $\left|\Delta S_{2 \Perp}\right|>\left|\Delta S_{2}\right|$. Например, при $k=1$, получим $\Delta S_{1}=1,4 K$, $\Delta S_{2}=1,3 \mathrm{~K}, \Delta S_{2 \mu}=1,9 \mathrm{~K}$. Обратно значение температуры термометрируемого объекта рассчитывается как полусумма обратных значений измеренной и зеркальной двухцветовых температур излучения [3]. Поэтому, погрешность расчета температуры объекта меньше погрешности для зеркальной двухцветовой температуры излучения примерно в 2 раза. Для указанных условий $\Delta T_{\text {дкпи }}=0,9 К(0,058 \%)$.

Относительная погрешность одноцветовой пирометрии излучения на $\lambda_{1}$ изменяется от $-0,336$ до $0,300 \%$ и определяется выражением [1]

$$
\delta T_{\lambda_{1}}=-\frac{\lambda_{1} \ln \left(\frac{\varepsilon_{\lambda_{1} \mathrm{H}}}{\varepsilon_{\lambda_{1}}(k)}\right)}{\lambda_{1} \ln \left(\frac{\varepsilon_{\lambda_{1} \mathrm{H}}}{\varepsilon_{\lambda_{1}}(k)}\right)+\frac{C_{2}}{T}} .
$$

Отсюда видно, что при $k<k_{\text {н }}, \delta T_{\lambda_{1}}<0$, так как $\varepsilon_{\lambda_{1}}<\varepsilon_{\lambda, \mathrm{H}}$, а при $k>k_{\mathrm{H}}, \delta T_{\lambda_{1}}>0$, так как $\varepsilon_{\lambda_{1}}>\varepsilon_{\lambda_{1} \mathrm{H}}$. Для одноцветовой Пирометрии излучения на $\lambda_{2}$ относительная погрешность определяется выражением, аналогичным (4). В указанных условиях эта погрешность изменяется от 0,456 до $-0,434 \%$. Зависимость $\delta T_{\lambda}=f(k)$ также отличается качественно от $\delta T_{\lambda_{1}}=f(k)$. Это объясняется тем, что при $k<k_{\mathrm{H}}, \delta T_{\lambda_{1}}>0$, так как $\varepsilon_{\lambda_{2}}>\varepsilon_{\lambda_{2} \mathrm{H}}$, а при $k>k_{\mathrm{H}}, \delta T_{\lambda_{2}}<0$, так как $\varepsilon_{\lambda}<\varepsilon_{\lambda_{\mathrm{H}}}$. Вследствие влияния длины рабочей волны $\left|\delta T_{\lambda}\right|>\left.\left.\right|^{2} \delta T_{\lambda_{2}}\right|^{\mathrm{H}^{\mathrm{H}}}$.

Погрешность пирометрии спектрального отношения пропорциональна отклонению коэффициента селективности излучения от настроечного , изменяется от $-3,013$ до 2,950 \% и определяется выражением [1]

$$
\delta T_{\mathrm{CO}}=-\frac{\lambda_{\text {экв }} \ln \left(\frac{k_{\mathrm{H}}}{k}\right)}{\lambda_{\text {эКв }} \ln \left(\frac{k_{\mathrm{H}}}{k}\right)+\frac{C_{2}}{T}} .
$$

При $k<k_{\mathrm{H}}, \delta T_{\mathrm{CO}}<0$, а при $k>k_{\mathrm{H}}, \delta T_{\mathrm{CO}}>0$.

От температуры зависят электромагнитные характеристики металлов и сплавов, которые, в свою очередь, определяют их спектральные распределения излучательной способности в инертной среде или вакууме. Например, для выбранных рабочих волн $\lambda_{1}$ и $\lambda_{2}$ спектральная излучательная способность вольфрама в вакууме при повышении его температуры от 1550 до 1650 К уменьшается соответственно на 0,0026 и 0,0022 , а коэффициент селективности - на 0,0005 [2]. В этих условиях погрешности ДКПИ и энергетической одноцветовой на $\lambda_{1}$ и $\lambda_{2}$, а также спектрального отношения термометрии на $\lambda_{\text {экв}}$, за счет связанных с температурой изменений излучательных характеристик, по модулю достигают соответственно 0,$031 ; 0,029 ; 0,033$ и $0,027 \%$. Эти погрешности пренебрежимо малы по сравнению с погрешностями, обусловленными оксидными пленами в обычной атмосфере. Поэтому, температурнозависимые составляющие можно не учитывать при расчете суммарных методических погрешностей. Селективность излучения металлов и их сплавов определяется воздействием множества случайных факторов, в том числе их химическим составом, количеством неметаллических включений, временной выдержкой, интенсивностью перемешивания и восстановительно-окислительных процессов. Поэтому «селективная» методическая погрешность подчиняется нормальному закону распределения. Имеющие для нормального распределения доверительную вероятность 0,68 средние квадратичные погрешности исследованных методов равны

$$
\begin{gathered}
\sigma_{\text {дкпи } k}=\frac{\left|\delta T_{\text {дкпи } k}\right|}{3}=\frac{0,069}{3}=0,023 \% ; \\
\sigma_{\lambda_{1} k}=\frac{\left|\delta T_{\lambda_{1} k}\right|}{3}=\frac{0,336}{3}=0,112 \% ; \\
\sigma_{\lambda_{2} k}=\frac{\left|\delta T_{\lambda_{2} k}\right|}{3}=\frac{0,456}{3} \approx 0,152 \% ; \\
\sigma_{\mathrm{CO} k}=\frac{\left|\delta T_{\mathrm{CO} k}\right|}{3}=\frac{3,013}{3} \approx 1,004 \% .
\end{gathered}
$$

При доверительной вероятности технических измерений, равной 0,95 , указанные погрешности соответственно равны

$$
\begin{gathered}
\delta T_{\text {дкпи } 0,95}=2 \sigma_{\text {дкпи } k}=0,05 \% ; \\
\delta T_{\lambda_{1} 0,95}=2 \sigma_{\lambda_{1} k}=0,22 \% ; \\
\delta T_{\lambda_{2} 0,95}=2 \sigma_{\lambda_{2} k}=0,30 \% ; \\
\delta T_{\mathrm{CO} 0,95}=2 \sigma_{\mathrm{CO} k}=2,01 \% .
\end{gathered}
$$

При учете остальных методических и инструментальных составляющих «селективные» методические погрешности классической термометрии, и прежде всего спектрального отношения, становятся недопустимыми для технических измерений температуры металла. Для качественного управления металлургическими процессами погрешность измерений температуры металлических сплавов обычно не должна превышать $1,0 \%$, а в случае, например, непрерывной разливки $-0,5 \%$.

Таким образом, в результате исследований установлены значительно более высокие метрологические характеристики, по сравнению с известными решениями, разработанного авторами метода двухцветовой компенсационной пирометрии излучения наиболее распространенных в металлургии и металлургии машиностроения железоуглеродистых сплавов со случайно изменяющейся в широких пределах селективностью спектральной излучательной способности. В исследованных условиях вызванные селективностью излучения методические погрешности двухцветовой компенсационной термометрии ниже погрешностей классической энергетической одноцветовой на $\lambda_{1}$ и $\lambda_{2}$, а также спектрального отношения пирометрии излучения металлических сплавов в видимой и ближней областях спектра в 4,4; 6,2 и 40,2 раза, соответственно. 
Цель работы - исследование влияния селективности излучения металлических сплавов на методические погрешности оптической термометрии. Для достижения поставленной цели в одинаковых условиях исследованы погрешности нового метода двухцветовой компенсационной, а также классической одноцветовой и спектрального отношения пирометрии излучения. Использованы спектральные методы исследований, основанные на полученных из термодинамических законов теплового излучения, в том числе законе Вина, пирометрические уравнения и теория случайных погрешностей. Доказаны значительно более высокие метрологические характеристики двухцветовой компенсационной пирометрии излучения по сравнению с классической термометрией в условиях случайно изменяющейся селективности из-

\section{SELECTIVITY INFLUENCE OF HEAT RADIATION OF METALL ALLOYS ON METHODICAL ERRORS OF THEIR CLASSICAL AND TWO-COLOUR COMPENSATING THERMOMETRY}

\section{Zhukov L.F., Petrenko D.A.}

Physics-Technological Institute of Metals and Alloys of NAS of Ukraine, 34/1, Vernadsky av., Kiev, 03142, Ukraine. References 3, figures 2.

Key words: temperature, metal alloys, two-colour compensating, energy one-colour and spectral ratio pyrometry, coefficient of selectivity of heat radiation, relative methodical error.

The objective of paper is to research selectivity influence of heat radiation of metal alloys on methodical errors of optical thermometry. To achieve the objective errors of new method of two-colour compensating, classical one-colour and spectral ratio thermometry were researched under the same conditions. Spectral methods of research, based on derived from thermodynamical laws, including лучения металлических сплавов.

\section{ЛИТЕРАТУРА}

1. Жуков Л.Ф., Петренко Д.А. Влияние температуры на методические погрешности двухцветовой компенсационной термометрии металлических сплавов // Процессы литья. - 2016. - № 6. - С. 47-53.

2. Шейндлин А.Е. Излучательные свойства твердых материалов: Справочник. // Под ред. А. Е. Шейндлина. - М: Энергия, 1974.

3. Жуков Л.Ф., Петренко Д.А., Корниенко А.Л. Двухцветовая компенсационная термометрия металлических сплавов и ее инструментальные погрешности // Процессы литья. - 2016. - № 5. - С. 48 - 58.

Wien's law, pyrometrical equations and theory of random errors were applied. Under conditions of randomly variable selectivity of heat radiation of metal alloys much more higher metrological characteristics of two-colour compensating pyrometry in comparison with classical thermometry were proved.

1. Zhukov L.F., Petrenko D.A. Temperature influence on methodical errors of two-colour compensating thermometry of metal alloys. Protsessy litya. 2016. № 6. P.47-53. (Rus.). 2. Zhukov L.F., Petrenko D.A., Kornienko A.L. Twocolour compensating thermometry of metal alloys and its instrumental errors. Protsessy litya. 2016. № 5. P.48-58. (Rus.).

3. Sheindlin A.E. Radiative properties of solid materials: Handbook. Moscow. Energia. 1974. 442 p. (Rus.).

Получено 07.02.2017 Received 07.02.2017 